\title{
An Energy Efficient Hybrid MAC Protocol with Dynamic Sleep-Based Scheduling for High Density IoT Networks
}

\author{
Thair. A. Al-Janabi, Member, IEEE, and Hamed. S. Al-Raweshidy, Senior, IEEE,
}

\begin{abstract}
Advances in the development of low powered sensors has meant they can now provide solutions to IoT networks that suffer from restricted power supply and a lack of resource facilities. In this paper, a hybrid TDMA-CSMA/CA MAC protocol has been proposed that efficiently utilises the energy of the nodes and dynamically adapts the sleep/wake-up periods according to the variance in the network loads. This hybrid protocol first schedules the TDMA time slots $\left(T D M A_{\text {slots }}\right)$, and then allocates each slot to a group of devices that compete for the medium using the CSMA/CA. This case is different from the traditional CSMA/CA-TDMA hybrid protocol, in which all the devices compete to access the channel, following which, each successful device is allocated an individual time slot. At the commencement of each superframe, the base station broadcasts a scheduler table, which includes network grouping information that is used by the IoT devices to categorise themselves into wake-up and sleep groups. To reduce the number of collisions or channel access failures, this information permits only one group to compete for each $T D M A_{\text {slot }}$. A three-dimensional Markov model is used to develop a per user stochastic behaviour for the proposed hybrid MAC protocol-based adaptable sleep mode. The simulation results demonstrate the effectiveness of the proposed protocol, which improves the network throughput and enhances energy conservation by $40 \%$ - $60 \%$ more than the IEEE 802.15.4based MAC protocol.
\end{abstract}

Index Terms-IEEE 802.15.4, Hybrid MAC protocol, PAN coordinator, IoT, Sleep mode, Scheduler table $(S T)$.

\section{INTRODUCTION}

$\mathbf{T}$ $\mathrm{HE}$ increased globalisation of information and communication technologies has transformed the world into the internet of things (IoT). The IoT refers to the network connectivity of physical or non-human things to the internet which, along with a level of artificial intelligence (AI), enables these things to collect and exchange data [1]. The IoT enables physical objects to see, hear, think and perform jobs by enabling them to communicate to share information and coordinate decisions [2]. Things, in the IoT, refer to a multitude of heterogeneous devices with attached sensors working across diverse applications. Moreover, wireless sensor networks (WSNs) are considered as an essential component of these networks [3]. Power consumption, however, is the primary restriction regarding IoT devices. Consequently, the

T. A. Al-Janabi is with the Department of Information and Communication Technology/ Middle Technical University, Iraq and H. S. Al-Raweshidy is with Wireless Networks and Communications Centre (WNCC)/ Department of Electronic and Computer Engineering College of Engineering, Design and Physical Sciences/ Brunel University London, London, UK e-mail: Thair.aljanabi@brunel.ac.uk ; Hamed.Al-Raweshidy@brunel.ac.uk

Manuscript received ; revised March 12, 2019
IEEE802.15.4 standard [4] for low power medium access control (MAC) and physical network specifications have been used with them to address insufficiency in the power supply [5].

In the IEEE 802.15.4 standard [4], a central controller known as the personal area network (PAN) coordinator oversees the construction, upgrading and monitoring across the entire network. Furthermore, the standard assigns two mechanisms, beacon-enabled and beaconless, to access the channel. The beacon-enabled mechanism uses a slotted carrier sense multiple access with a collision avoidance mechanism (CSMA/CA) to share the medium. In this mechanism, the slot margins of each device are aligned with those margins of the PAN coordinator using beacons and conversely, in the beaconless mechanism, a simpler unslotted CSMA/CA is applied. The beacons contain information used to describe the construction of superframes and synchronise the devices in the transmission range to recognise the PAN coordinator. The superframe comprises active and inactive portions, with former consisting of the contention access period $(\mathrm{CP})$ and the contention free period (CFP). Throughout the inactive portion, the device does not interact with its coordinator and may move to a low power mode to conserve energy [4].

In general, the energy consumption of the network is highly influenced by the selection of the MAC protocol [6], which is divided into three main categories: contention free protocol, contention-based protocol and hybrid protocol [7]. Regarding the contention free protocol, in IoT networks it usually uses the time division multiple access (TDMA) mechanism to control the channel [8]. TDMA is a channel access technique that authorises a set of devices to access a certain carrier frequency without intervention. Furthermore, it is useful for extending the battery life of the devices and reducing the number of collisions. However, TDMA has some drawbacks, for example, it is difficult to adapt to variant network loads, i.e. low or high, leading to either inefficient channel utilisation or limited network scalability. Another drawback is that the transmission is delayed when the beacon interval is increased due to the fixed size of the beacon intervals [9].

CSMA/CA is a contention-based mechanism used to share the medium randomly between the IoT devices [10]. This random technique is applied to low power devices such as those associated with IEEE 802.11 [11] or IEEE 802.15.4 [12]. However, contention based protocols suffer from high congestion associated with either a high density of IoT devices or a hidden node problem [9]. This high congestion leads to 
an increase in the number of retransmissions, which affects the overall network performance in terms of channel utilisation and power consumption. A hybrid CSMA/CA-TDMA protocol is, therefore, useful for avoiding the weaknesses of the above protocols and combining their strengths to enhance channel utilisation, whilst also reducing power consumption in wireless networks [13]. However, the performance of the hybrid protocol still seems to suffers from enormous energy depletion in high-density networks . Thus, adaptable scheduling, involving dynamic switching between sleep and wake-up periods, is required to accomplish superior channel access in such networks.

In this study, an on-demand sleep/wakeup extension is proposed to the IEEE 802.15.4 standard to deal with the abovementioned IoT issues. The sleeping periods $(L S)$ are dynamically adapted according to the network density, collision status and channel failure, thus leading to efficient throughput and power savings as well as high channel utilisation. The base station (BS) arranges and schedules the TDMA $A_{\text {slots }}$ and the scheduling table (ST), whereas each individual device, to save energy, has to calculate its $L S_{i}$ and switch to a sleep/wakeup period. The information in the ST message enables a certain group of devices to compete for entering the transmission period. In contrast, other groups are forced to enter the sleep mode each with a different calculated $L S_{i}$. However, when the network load is low or there is no collision, then all the devices can continue accessing the channel at any TDMA slot after a competition to access the channel using CSMA/CA. Moreover, the devices move into sleep mode only when the medium experiences a high collision rate or channel failure. This has the advantage of avoiding a costly channel access pattern by reducing the number of collisions occurring due to a high density of devices. Furthermore, the proposed hybrid protocolbased adaptable sleep model can be theoretically displayed using a three-dimensional Markov chain and then compared to the original IEEE 802.15.4. Hence, this paper makes the following contributions to current knowledge:

- It proposes a dynamic hybrid MAC protocol for high density IoT communications with a scheduler sleep/wakeup mode called HSW-802.15.4. This mode has been designed to be activated only after the device experiences a high collision rate and/or channel failure situation.

- It develops a frame structure scheme for the proposed protocol, which offers enhanced exploitation of TDM $A_{\text {slot }}$ and compatibility with the IEEE 802.15 .4 standard.

- It provides a comprehensive analytical evaluation using a three-dimensional Markov model that predicts the performance of the proposed hybrid protocol-based dynamic sleep mode.

- It evaluates and verifies the performance of the proposed model for small and high-density networks by comparing it with the traditional IEEE 802.15.4 standard.

The remainder of this paper is structured as follows. Section II briefly discusses previous research related to the IoT networks, whilst Section III describes the formulation of the problem behind the development of the proposed HSW-802.15.4. The frame structure and the methodology for the proposed protocol are provided in Section IV. Section V briefly discusses the proposed IEEE 802.15.4 based dynamic sleep mode and presents an analytical model for a three-dimensional Markov chain compatible with the proposed model for calculation of the network metrics. Section VI validates the proposed protocol by comparing the results with the existing IEEE 802.15.4 standard. The numerical results are presented and discussed in Section VII, whilst final conclusions are presented in Section VIII.

\section{RELATED RESEARCH}

This literature divides the MAC studies into two categories: the analytical-based studies and the simulation-based ones. Furthermore, it splits the former based on a Markov mode into two classes: those that mimic and evaluate the performances and properties of the IEEE 802.15.4 standard, and those that attempt to improve the performance of this standard.

\section{A. Analytical-Based Mac Protocols}

Many studies have been aimed at assessing and understanding the analytical MAC model of the IEEE 802.15.4 standard. These studies have attempted to mimic aspects and mechanisms widely used by the IoT and WSN devices to investigate and identify the properties and performances of those networks. Most of the analytical models for the IEEE 802.15.4 standard are built according to the Markov model, presented by Bianchi [11] for the IEEE 802.11 standard. That is, Bianchi designed a Markov chain model that mimics the functionalities of the IEEE 802.11 standard for ideal channel conditions and saturated networks. In this subsection the analytical studies based on a Markov mode is split into two categories: those that mimic and evaluate the performances and properties of the IEEE 802.15.4 standard, and those that attempt to improve the performances of the IEEE 802.15.4 standard.

1) Performance Evaluation Studies: From a performance evaluation perspective, a comprehensive Markov chain model and analysis for the IEEE 802.15.4 [4] MAC and PHY layers has been conducted by many authors [9], [12], [14]-[20]. For example, Pollin et al. [12] proposed a two-dimensional Markov model, with an extension of saturated and unsaturated mechanisms for the slotted CSMA/CA mechanism that mimics the MAC behaviour of the IEEE 802.15.4. The performance of the IEEE 802.15.4 standard in terms of power depletion and throughput was also analysed. In contrast, Sahoo et al. [15] proposed a modified model including retransmission with determinate retry limits for the IEEE 802.15.4. However, it appears that there was an inexact match between the analytical and the IEEE standard results for both energy depletion and throughput. Park et al. [16] proposed a generalised analytical model of IEEE 802.15.4 with retry limits and acknowledgements under unsaturated star networks. This was an accurate Markov model that could evaluate the performance metrics in terms of delay, reliability and energy depletion.

On the other hand, Zayani et al. [17] developed an IEEE 802.15.4 model that takes into consideration the functionalities of this standard on both the PHY and MAC layers. On the 
PHY layer, the model is similar to the mathematical framework used by Zuniga and Krishnamachari [18] for calculating link unreliability. Moreover, the MAC layer is inspired from an improved Markov model proposed by Park et al. [16]. This model considers the effect of underlying packet discards at either the PHY or MAC layers [17]. Performance estimation in all the above studies has been aimed at providing optimal Markov models for the CSMA/CA mechanism that mimic the behaviour of IEEE 802.15.4 standard; however, they do not seem to have involved taking the sleep/wakeup mode or any other improvement into consideration.

2) Performance Improvement Studies: To improve energy preservation and the performance of the IEEE 802.15.4 standard, several researchers [21]-[27] have proposed models with periodic sleep or idle periods, and device contention, as perfect solutions. Misic et al. [21], for example, suggested an M/G/1/K model of a beacon-enabled IEEE 802.15.4 with a sleep mechanism. With the proposed mechanism, it is assumed that a device turns to sleep for a specific amount of time when there are no packets in its buffer [21]. Alternatively, Chiasserini et al. [22], considered the performance of sleeping devices in WSNs; however, they did not focus on the performance evaluation of the IEEE 802.15.4 MAC layer.

From the performance evaluation aspect, Ghazvini et al. [23] suggested an energy efficient MAC protocol, based on IEEE 802.15.4, for WSNs that reduces energy depletion and network collisions by permitting devices to move between sleep and listening modes during the active period, when they have no packets to transmit. After that, Xiao et al. [24] also proposed a Markov model that took into consideration the sleep-based mode of the beacon-enabled IEEE 802.15.4 standard. Jurdak et al. [25], in contrast, implemented the non-beacon IEEE 802.15.4- based RFID devices. To conserve energy, a device remains in sleep mode, if it has no packet to send or the RFID tag has not received any data. Khanafer et al. [26] suggested a method that saves energy by forcing some devices to move into sleep mode after successful communication. Furthermore, Xiao et al. [27] proposed an energy efficient MAC protocol with a sleep enabling mode based on the length of the device queue. However, all these studies focused only on the contention-based channel access, and not on network density, the numbers of collisions or even channel failure.

Consequently, the performance of the hybrid IEEE 802.15.4 MAC protocol has also been studied by [28], [29]. The research conducted in [28] offered a general Markov model that takes into consideration both the slotted CSMA/CA and GTS in a heterogeneous network set-up and non-saturated conditions. The authors in [29] focused on reducing the collisions caused by the hidden node problem in IEEE 802.15.4 networks using the GTS slots. Recently, Al-Janabi et al. [30] introduced a solution that expands network scalability and prolongs the lifetime of the IoT network protocol by developing a scheduling sleep/wake-up model based on artificial intelligence (AI). In addition, Ayadi et al. [31] provided two methods that aim to better manage the energy exhausted by the WSNs. The first method concerned efficiently identifying the energy depleted by the device in its various transmission stages, whilst the other method provided an approach that based on the residual energy of the devices to identify their most compatible duty cycles. Although, both of the above protocols provided energy efficient solution for the IoT network, they did not consider the high density of the IoT network.

\section{B. Simulation-Based Mac Protocols}

It is generally agreed that MAC protocols are the simplest techniques in terms of system set-up and implementation, some of which are described below.

Amongst the most basic MAC protocols are the random access protocols, such as ALOHA and slotted-ALOHA, stated by [32], [33], respectively. In these protocols, the devices either send the packet as soon as it is generated or start the transmission at the commencement of the following slot. The key downside of these random-based protocols is the high rate of collisions, which restricts the value of the throughput and eliminates the utilisation of the channel bandwidth [33]. CSMA protocols are considered an advancement toward decreasing the collisions suffered by ALOHA methods [34]. However, these protocols do not significantly diminish the collisions and there can be degradation in the throughput caused by the hidden and collided node problems.

Hybrid MAC protocols that associate CSMA with TDMA, which are described by Rhee et al. in [35] and Zhang et al. in [36], act as a CSMA with a light contention state and change to the TDMA MAC time sort procedure at a higher contention state. On the other hand, Salajegheh et al. in [37] proposed a hybrid MAC (HyMAC) that combines CSMA with both TDMA and FDMA. In a comparable manner, other hybrid MAC protocols have been proposed in [38], [39]. In those protocols, the devices combine CDMA with TDMA and utilise CSMA to send their request for a bandwidth message to reserve time slots and codes for their transmission successfully. Another hybrid MAC protocol, called zebra MAC (ZMAC), is proposed by Rhee in [35]. The hybrid technique of this protocol works as CSMA in a low network traffic and switches to TDMA when it is high. However, if the devices require more than one-time slot, they can try to exploit other neighbours' unutilised time slots. To exploit other time slots, the device initiates a long enough random backoff period and starts its transmission at the end of this period, but the device is not able to start its transmission if the primary user needs its own time slot. Moreover, ZMAC is required to implement TDMA scheduling in a periodic manner to recover the deviation in the time clock among the devices.

A scalable Hybrid CSMAMAC protocol is suggested in [1], which works by splitting each frame period into four portions: notification period (NP); contention only period (COP); announcement period (AP); and transmission only period (TOP). Throughout the COP, the devices with data to send employ the p-persistent CSMA mechanism to transmit the request to send (RTS) packet to the BS and those that succeed in doing so, are allocated time slots in the TOP to transmit their data. This hybrid MAC protocol is improved by Liu et al. in [40] by the implementation of the fairness and quality of service (QoS) concepts. The improved protocol categorises the devices into different classes and permits the devices to pick up the 
contention probability rendering to their observed throughput and urgency. However, both the above protocols proposed in [1], [40] suffer from supplementary energy depletion caused by the requirement for the contention between devices during the COP.

Despite the above hybrid protocols trying to be more scalable, they are still not adequate for deployment in most IoT applications. Also, they still seem to suffer from a higher number of collisions and channel failure in saturated and highdensity networks due to their failure to include a sleep/wakeup mechanism in their operation. Consequently, congestion in the IoT network increases as the network density rises. Moreover, the hybrid protocols-based on FDMA and CDMA have the issues of high hardware expenses and high complexity, respectively. Hence, the hybrid MAC protocols based on both TDMA and CSMA are the most encouraging from the perspective of IoT applications. Accordingly, the proposed HSW-802.15.4 protocol provides an amenable hybrid TDMACSMA/CA MAC mechanism with a dynamic sleep/wake-up extension that efficiently accesses the channel with both low and high-density networks. The proposed protocol tunes up the sleep/wake-up periods by forcing groups of devices to sleep for various periods once they experience a collision or channel failure. As a result, the proposed model works by reducing the hidden node problem, and thus reduces the probability of collisions, thereby leading to an improvement in the performance of the network in terms of power conservation, channel utilisation, and throughput.

\section{PROBLEM Formulation}

IoT devices generally suffer from two types of problems, namely: device resource restriction and protocol design.

Regarding device resource restriction, IoT devices are all severely constrained in terms of power supply, communication capability and memory units. In fact, power consumption is the primary restriction placed upon them. Moreover, the vast majority are not rechargeable because they are usually designed in a small-sized system that constrains the capacity of the battery and memory. Additionally, the periodic battery replacement for those devices is infeasible due to the device deployment in a vital environment and the large number of IoT devices. Furthermore, the complex computations that necessitate unique processing capabilities and extended memory units require massive power resources and thus, dramatically reduce the overall lifespan of the IoT network. The strict resource-constrained situations in which IoT devices operate make energy conservation a primary factor to be considered when undertaking protocol design in such networks.

Regarding the protocol design problem, the MAC protocol responsibility should be in handling the channel access mechanisms, failure control, and validation of the superframes. However, MAC protocols such as CSMA/CA and TDMA usually focus on some network parameters and ignore others. For example, TDMA takes into consideration energy saving and neglects throughput, delay and channel utilisation. Furthermore, with TDMA, it is difficult to adapt the channel to the variations in the network density, whether this is low or high load, thus leading to either inefficient channel utilisation or limited network scalability. On the other hand, the hybrid MAC protocol is useful for addressing the weaknesses of the above protocols and also combines their strengths. However, it cannot ensure efficient energy saving in a high-density network. Hence, a dynamic MAC protocol that minimises the energy consumed by IoT devices during their connection process to the Telecom is essential and needs to be designed. Furthermore, extended periods of sleeping devices and the use of energy efficient techniques can all help to extend the battery life of these devices.

Accordingly, in this work, an effective power saving structure using a dynamic scheduling MAC technique is proposed. This technique dictates the task to be achieved at a particular time and obliges groups of devices to sleep. Furthermore, the scheduling sleeping technique is adapted according to conditions, such as high collision or channel failure, to determine which group of devices will be active in a certain $T D M A_{\text {slot }}$. The proposed protocol will accomplish superior channel access enhancement in a high density network and extend the lifespan of IoT devices.

\section{Proposed Scheduler Hybrid MAC Protocol}

The novelty of this work is represented by suggesting a new sleep/wake-up extension to the mechanism of the IEEE 802.15.4 standard that forces groups of devices, when the network experiences high collisions or channel failure, to sleep mode with different sleep periods. Therefore, this extension makes the proposed HSW-802.15.4 MAC work efficiently with both high and small density IoT networks. However, to provide an analytical model for the proposed protocol, a three dimensions Markov model has been presented that includes the new sleep/wake-up mode in its operation. Further details about the Markov model are presented by [14], [17].

Power consumption is a vital issue for battery power supported devices in IoT and WSNs. To address this problem, an effective scheduling message, known as the scheduling table (ST) (see Figure 1), has been proposed to force groups of them into sleep mode. The proposed scheduling method is extremely efficient in terms of energy conservation, especially for battery-powered devices. It selects the mission to be performed at a given moment by determining which group of devices will be active in each $T D M A_{\text {slot }}$, and by allocating physical layer resources. in this section, the structure of the proposed protocol in terms of network architecture, frame structure and methodology is discussed.

\section{A. Network Architecture}

A star wireless personal area network with a single-hop is assumed to be used in the simulation. It is composed of a fixed number $(\mathrm{N})$ of devices and a personal area network (PAN) coordinator. Furthermore, it is assumed that, as a highdensity network, each device always has a packet available for sending, which means it is working in a saturated area. The devices also exhibit location awareness pro-actively during the device installation process without any the device cooperation, and then can send their location along with their identification 


\begin{tabular}{|c|c|c|}
\hline \multicolumn{3}{|c|}{ The structure of the scheduling table (ST) } \\
\hline Subgroups & Groups of Nodes' ID & Sleep periods \\
\hline SG1 & $I D_{1}, I D_{2}, \ldots \ldots \ldots \ldots \ldots . ., I D_{i}$ & K1 slots \\
\hline SG2 & $I D_{i+1}, I D_{i+2}, \ldots \ldots \ldots . ., I D_{j}$ & K2 slots \\
\hline 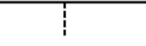 & : & 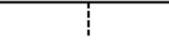 \\
\hline SGNG & $|\mathrm{DL}+1,| \mathrm{DL}+2, \ldots \ldots \ldots \ldots . ., \mathrm{IDN}$ & KNG slots \\
\hline
\end{tabular}

Fig. 1: The structure of the proposed scheduling table $S T$

address (ID) to the PAN coordinator to be utilised in avoiding the hidden node problem.

In the proposed protocol, the PAN coordinator divides the network devices into multiple subgroups $\left(S G_{i}\right)$ according to the ID and location of the device. At the commencement of each superframe, the coordinator sends the ST message as a notification during the beacon period. As Figure 1 shows, the ST message provides information about the number of subgroups (NG) and the IDs of the devices belonging to each $S G_{i}$. Additionally, it contains information about the sleep/wake-up times for each $S G$, which is used to conserve energy by specifying the maximum number of $T D M A_{\text {slots }}$ each device should have. These are known as $K i T D M A_{\text {slots }}$.

The KiTDMA $A_{\text {slots }}$ for each $S G_{i}$ are chosen by the coordinator according to device priority, so that devices at a $S G_{i}$ with higher priority are allocated fewer KiTDMA $A_{\text {slots }}$ than other subgroups. The ST message is thus sent after the beacon (See Figure 2) and used to schedule devices to enter a longer sleep mode, rather than a longer contention period (CP). The length of the ST message is constant and equivalent to multiple messages according to the network requirements and node density; however, each device needs to be aware of the size of the ST message. During the CP, the devices in the specified $S G_{i}$ are eligible to compete using the CSMA/CA method, to obtain access to the channel and transmit data during $T D M A_{\text {slots }}$, also known as transmission periods $\left(T P_{\text {transmission }}\right)$. However, when the network does not experience any channel collisions or failure, all the other devices can compete and obtain access to the channel as a normal IEEE 802.15.4, without an adaptable sleep mode.

\section{B. Frame Structure}

In this study, a hybrid MAC protocol is proposed in which a specified group of devices are permitted to enter the CP at a specific $T D M A_{\text {slot }}$, rather than all the devices. Figure 2 shows the modified superframe structure for the proposed model, which is compatible with the traditional IEEE 802.15.4. As such, the proposed hybrid protocol can significantly reduce network collisions and improve performance. It uses a superframe architecture where network beacons are periodically broadcast by the PAN coordinator to bind each superframe. The network beacons are used to describe the architecture of the superframes, and to synchronise as well as scheduling the attached devices by using the ST message (See Figure 3-a).

The superframe of the proposed protocol is, firstly, divided into 16 equal sized $T D M A_{\text {slots }}$ by the PAN coordinator, in a

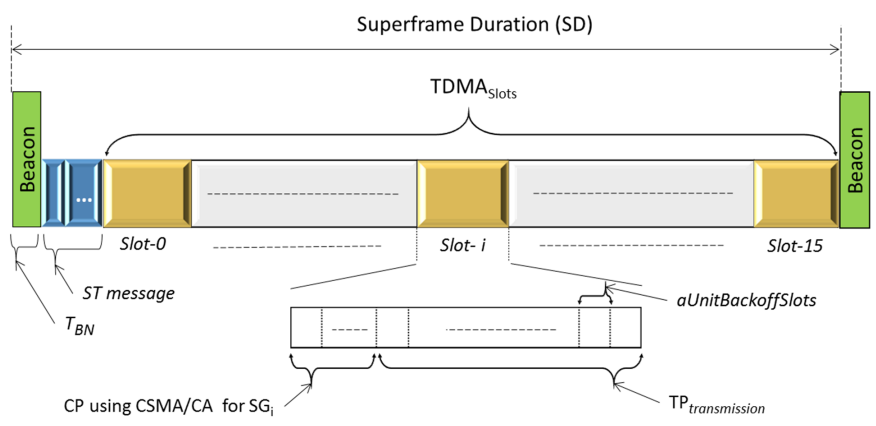

Fig. 2: The proposed superframe structure that shows the $C P$ and $T P$ periods while $T D M A_{\text {slots }}=16$

similar way to the IEEE 802.15.4 standard. Each TDM $A_{\text {slot }}$ is allocated to a specific $S G_{i}$. A device that wishes to access the channel can only compete with other devices in the same $S G_{i}$ using a CSMA/CA mechanism (See Figure 3-a). However, there is an exception to this rule, which is that the device can enter the contention period with other subgroups when the channel has not suffered from any previous failure or collision. This mechanism has the dual advantage of offering efficient utilisation of the channel in low-density networks, and reducing the number of collisions by following the ST message with high-density networks.

The present IEEE 802.15.4 protocol is based on a simple time unit called a UnitBackoffslots period (BP) and three random based variables: backoff exponent (BE), contention window (CW), and number of backoff stages (NBF). The proposed protocol introduces other substantial variables, which are the length of sleep period $\left(L S_{i}\right)$, and the number of $S G_{i}$ of the devices $(N G)$. As shown in Figure 3-b, after receiving the Beacon and the ST message from the PAN coordinator and agreeing with the proposed sleep/wake-up mode (see Section V), the device either immediately starts sending its data or follows the sleep mode, and then starts sending its data. Furthermore, each device has to calculate its sleep period continuously by utilising the information from the ST message.

\section{Methodology}

The proposed protocol is designed according to the specifications of IoT networks and the transmission process for the proposed HSW-802.15.4 protocol is shown in Figure 3c. In the proposed protocol, the MAC layer firstly initialises the following variables: contention window, backoff exponent $(\mathrm{BE}=\mathrm{MinBE})$, macMaxCSMABackoff $(M=2)$, number of backoffs counter $(N B F=0)$, maximum frame retries $(R=3)$, retransmission counter $(r=0)$, maximum number of CCAs $\left(C_{\text {Max }}=2\right)$, CCAs counter $(i=0)$, and number of $S G=N G$.

The device then waits for a random backoff number (BF) in the range $\left[0 ; 2^{B E}-1\right]$ multiplied by the time of the BP. When the backoff counter reaches zero, the device senses the channel using clear channel assessment (CCA). If two successive CCAs are idle, the device initiates the transmission, whilst if is busy, the MAC layer will increase the value of both 


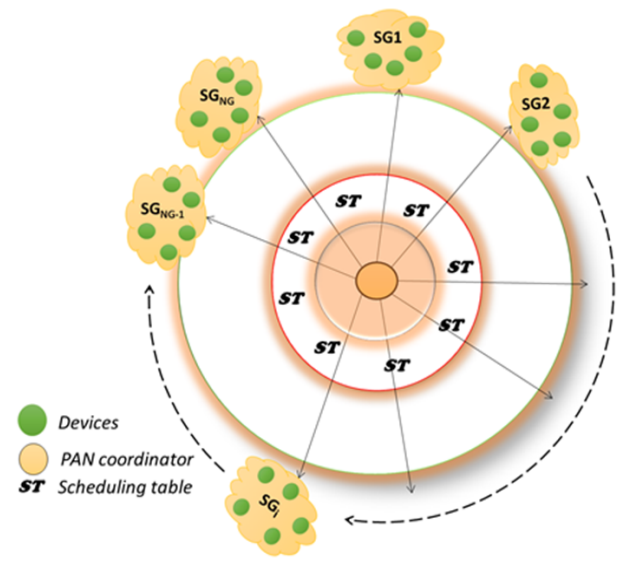

Figure (a)

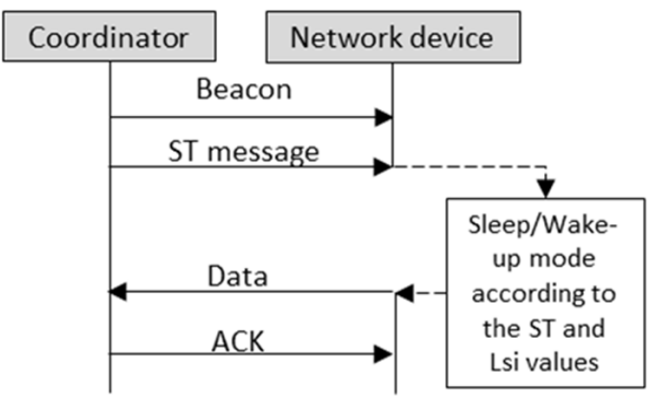

Figure (b)

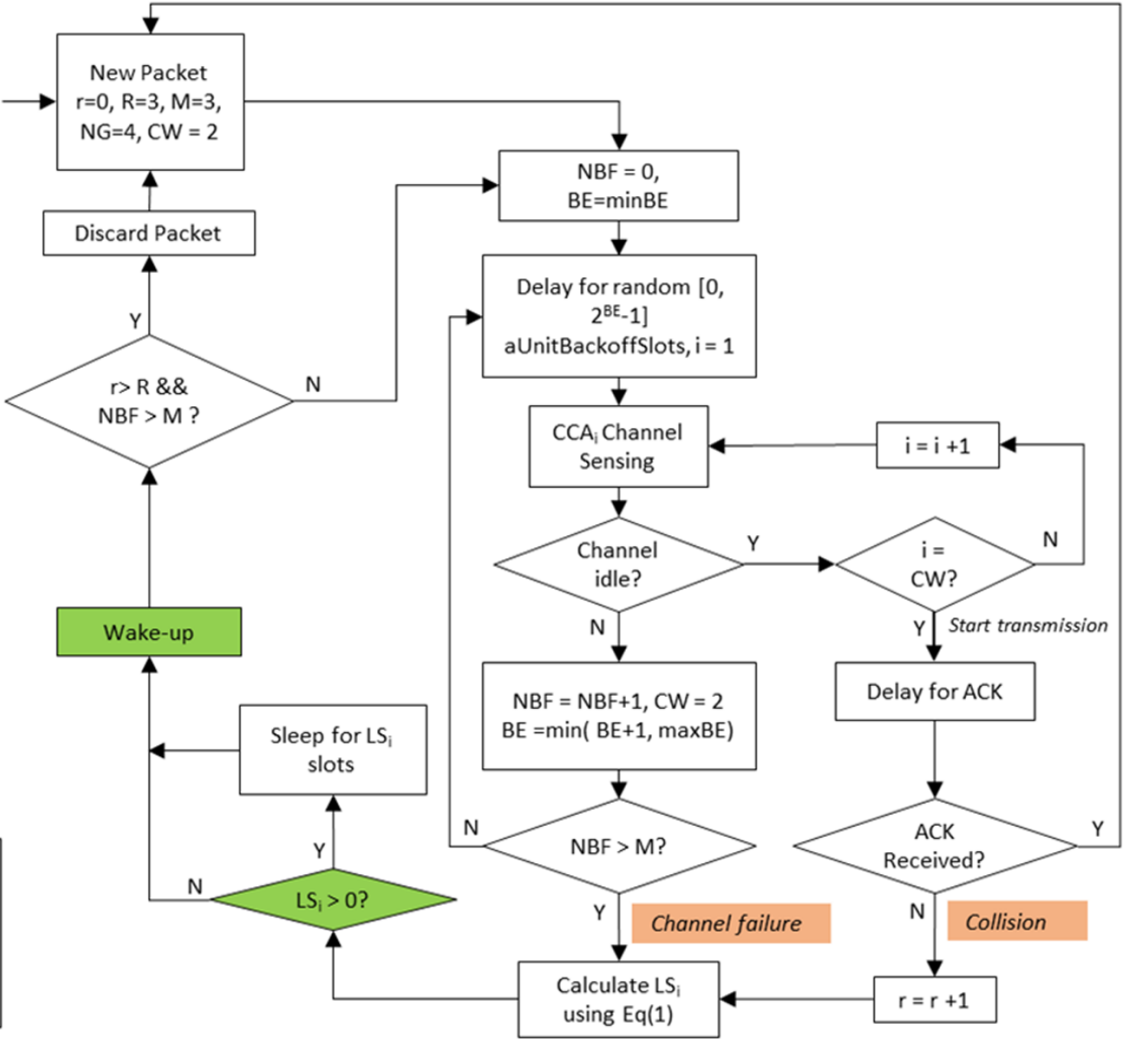

Figure (c)

Fig. 3: a- Network star topology with $S G_{i} s$, b- The overall communication scenario for a device using HSW-802.15.4, cTransmission process for the proposed HSW-802.15.4

$\mathrm{NBF}$ and $\mathrm{BE}$ by one up to a maximum value of $M$ and MaxBE respectively. If the channel access is successful, the device starts transferring its packet and waits for acknowledgement (ACK). If the device receives the ACK, the transmission has succeeded and if not, a collision is detected and the devices involved should increase the variable $r$ up to the value $R$. If the channel experiences a channel failure or a collision, the device calculates the length of the sleep period $L S_{i}$ and either continues trying to access the channel if it belongs to a permitted $S G_{i}$ and $L S_{i}=0$ or turns to sleep mode, according to the ST and the calculated $L S_{i}$ (see equation 1). After the $L S_{i}$ period, the device, depending on its NBF and $r$, decides either to discard its packet and generate a new one or to retransmit its packet by reinitialising the $\mathrm{NBF}=0, \mathrm{BE}$ $=\min B E, r=0$, and $i=0$. The parameters used for the proposed protocol are listed in Table I, which are the same as the default IEEE 802.15.4 MAC parameters.

\section{Analytical Model and Notations}

This section describes the proposed HSW-802.15.4 MAC protocol-based schedule sleep extension. The main aim of this protocol is to save power and prolong the overall lifespan of the IoT network. The energy consumption of the IEEE 802.15.4 standard is primarily influenced by the probability of channel access failure or collisions, and MAC parameters.
Hence, the proposed HSW-802.15.4 protocol employs a strategy to force some groups of nodes to sleep for a calculated $L S_{i}$ period, whenever there are channel collisions or channel failure.

Using this method, the proposed protocol becomes more adaptable and flexible as it dynamically adapts the sleep nodes, working with both high and low-density networks. The behaviour of this new adaptable protocol can be easily observed in a high-density network by allowing more nodes to sleep with different periods. The main benefit of this lies in a substantial reduction in the probability of channel failure and collisions. The following subsections explain the sleep period calculation used by each device, and the three-dimension Markov model which is based on a dynamic sleep mode. Additionally, the influence of performance metrics and MAC parameters on overall network operations are also analysed. Table III in Appendix A summarises the notation used in this paper.

\section{A. Dynamic Sleep/Wake-up Period Calculation}

The grouping method that divides the nodes into $S G_{s}$, whilst dynamically allowing the $S G_{s}$ to calculate and enter the Sleep/Wake-up periods, is described. In each specified $T D M A_{\text {slot }}$, only one $S G$ is permitted to compete for channel access (See Figure 4). The aim of this technique is to distribute 
TDMA slots (Active Period)

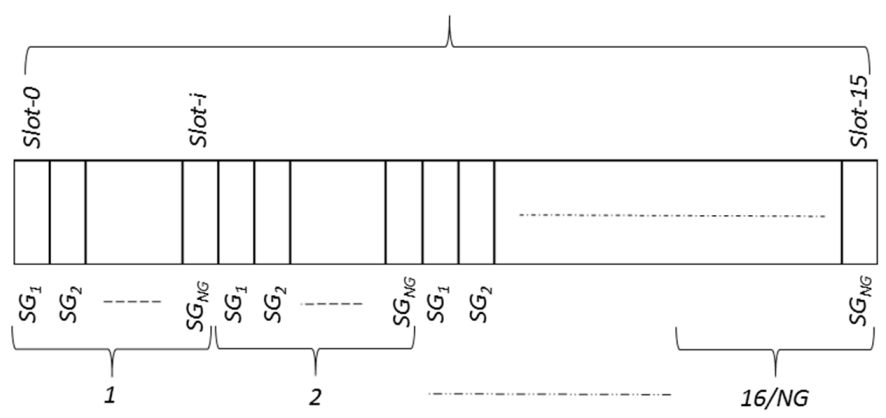

Fig. 4: Subgroups separation among time slots

the active nodes evenly among the $T D M A_{\text {slots }}$ and reduce the power consumed by each node as it attempts to access the channel. This is because, depending on the node density in the network, a collision may be more likely to occur at one $T D M A_{\text {slot }}$ than at others. Hence, when the device in subgroup $\left(S G_{i}\right)$ experiences a channel failure or collision, it calculates the length of its sleep period $\left(L S_{i}\right)$ as follows:

$$
L S_{i}= \begin{cases}Y_{i} \times N_{B P} & \text { if } Y_{i} \leq K_{i} \\ K_{i} \times N_{B P} & \text { if } Y_{i}>K_{i}\end{cases}
$$

where, $K_{i}$ refers to the number of sleeping TDMA slots allocated to the $S G_{i}$ that is initially identified by the PAN coordinator (see Figure 1), while $Y_{i}$ is calculated as

$$
Y_{i}=\mid S G_{i}-\left(\left(\text { Slot }_{\text {seq }} \bmod N G\right)+1\right) \mid
$$

where, $S G_{i}$ is the subgroup $i$ of devices to which the current device belongs. $N_{B P}$ refers to the number of BPs in each $T D M A_{\text {slot }}$. Slot $_{\text {seq }}$ is the sequence of the current TDMA $A_{\text {slot }}$ and lies between 0 and 15. NG represents the number of subgroups. Furthermore, the ratio of the active and sleep nodes is adjusted by adding the calculated number of sleep $T D M A_{\text {slots }}$ to the model based on $L S_{i}$.

The role of $L S_{i}$ is to decide whether the device that has experienced a collision or channel failure, turns to sleep, if $L S_{i}>0$ or continues its attempt to access the channel with the same $T D M A_{\text {slot }}$. In this case, the energy required to access the channel for the whole network is reduced, because some of the devices move to sleep mode, each for a different period, according to its calculated $L S_{i}$. It is worth noting that the sleep period identification process is similar to the random process used to identify the BF, which depends on the previous stage only and not on any others. In fact, the only difference between $\mathrm{BF}$ and $L S_{i}$ is that the former is selected randomly, whilst the value of the latter is calculated according to Equation (1).

However, as presented in Table II, the device with the proposed protocol, especially in a small density network, has to consume an additional small amount of energy for the purpose of receiving the ST message at the commencement of each superframe. This message is essential in predicting the $L S_{i}$ period, while it is not applicable for the case of the IEEE 802.15.4 standard.
TABLE I: The parameters used by the HSW-802.15.4

\begin{tabular}{|l|l|}
\hline Parameters & Values \\
\hline Number of backoff stages $(N B F)$ & $0 \ldots 5$ \\
\hline MinBE and MaxBE & 3 and 5, respectively \\
\hline Backoff Exponent $(B E)$ & $0 \ldots 3$ \\
\hline Contention period $(C W)$ & 2 \\
\hline Number of sleep stages $\left(K_{i}\right)$ & $0 \ldots N G$ \\
\hline Number of $S G_{i}(N G)$ & $1 \ldots 16$ \\
\hline Maximum frame retransmission $(R)$ & 3 \\
\hline Total packet size $\left(P C K_{L}\right)$ & 127 byte \\
\hline Data Rate $(D R)$ & $250 \mathrm{Kbit} / \mathrm{sec}$ \\
\hline$T D M A_{\text {slot }} i$ length & $60 \mathrm{~ms}$ \\
\hline Transmission power $\left(P W_{t x}\right)$ & $30 \mathrm{~mW}$ \\
\hline Receiving power $\left(P W_{R x}\right)$ & $40 \mathrm{~mW}$ \\
\hline Idle power $\left(P W_{i d l e}\right)$ & $0.8 \mathrm{~mW}$ \\
\hline Channel sensing power $\left(P W_{C C A}\right)$ & $40 \mathrm{~mW}$ \\
\hline Sleep power $\left(P W_{S L}\right)$ & $0.16 \mu \mathrm{W}$ \\
\hline
\end{tabular}

TABLE II: Performance comparison between HSW-802.15.4

\begin{tabular}{|c|c|c|}
\hline Type & Small density network & High Density network \\
\hline IEEE 802.15.4 & $\begin{array}{l}\text { Performs efficiently in } \\
\text { small area networks }\end{array}$ & $\begin{array}{c}\text { Inactive with the } \\
\text { high density networks }\end{array}$ \\
\hline HSW-802.15.4 & $\begin{array}{l}\text { A small amount of } \\
\text { extra energy is } \\
\text { consumed due to the } \\
\text { reception of the ST } \\
\text { message. However, it } \\
\text { still performs normally } \\
\text { as the IEEE 802.15.4 } \\
\text { after receiving } \\
\text { the ST message }\end{array}$ & $\begin{array}{l}\text { Works efficiently in } \\
\text { high density networks; } \\
\text { therefore, it is suitable } \\
\text { for the future IoT } \\
\text { with higher expected } \\
\text { number of devices }\end{array}$ \\
\hline
\end{tabular}
and IEEE 802.15.4 in small and high density networks

\section{B. Markov Model}

This subsection describes the analytical model for the proposed adaptive hybrid MAC protocol-based synchronised sleep mode. For the purposes of the analysis, a saturated network with beacon enabled and retry restrictions for each packet transmission is considered. The behaviour of the proposed HSW-802.15.4-based schedule sleep is modelled using a Markov model that takes into consideration the probabilities of collision and failure. Figure 5 shows the Markov modelbased sleep mechanism of the HSW-802.15.4.

The analysis is split into two distinct steps, the aim of which is to identify a set of equations that exclusively describes the functionality of the network. During the first step, the actions of a single device are modelled using a three-dimensional Markov chain to obtain the stationary probability $(\Theta)$ of the device attempting to access the channel for the first $C C A$ within a given time slot. The per user Markov chains are then coupled to derive an extra set of equations to solve the system model. A significant assumption made during this 


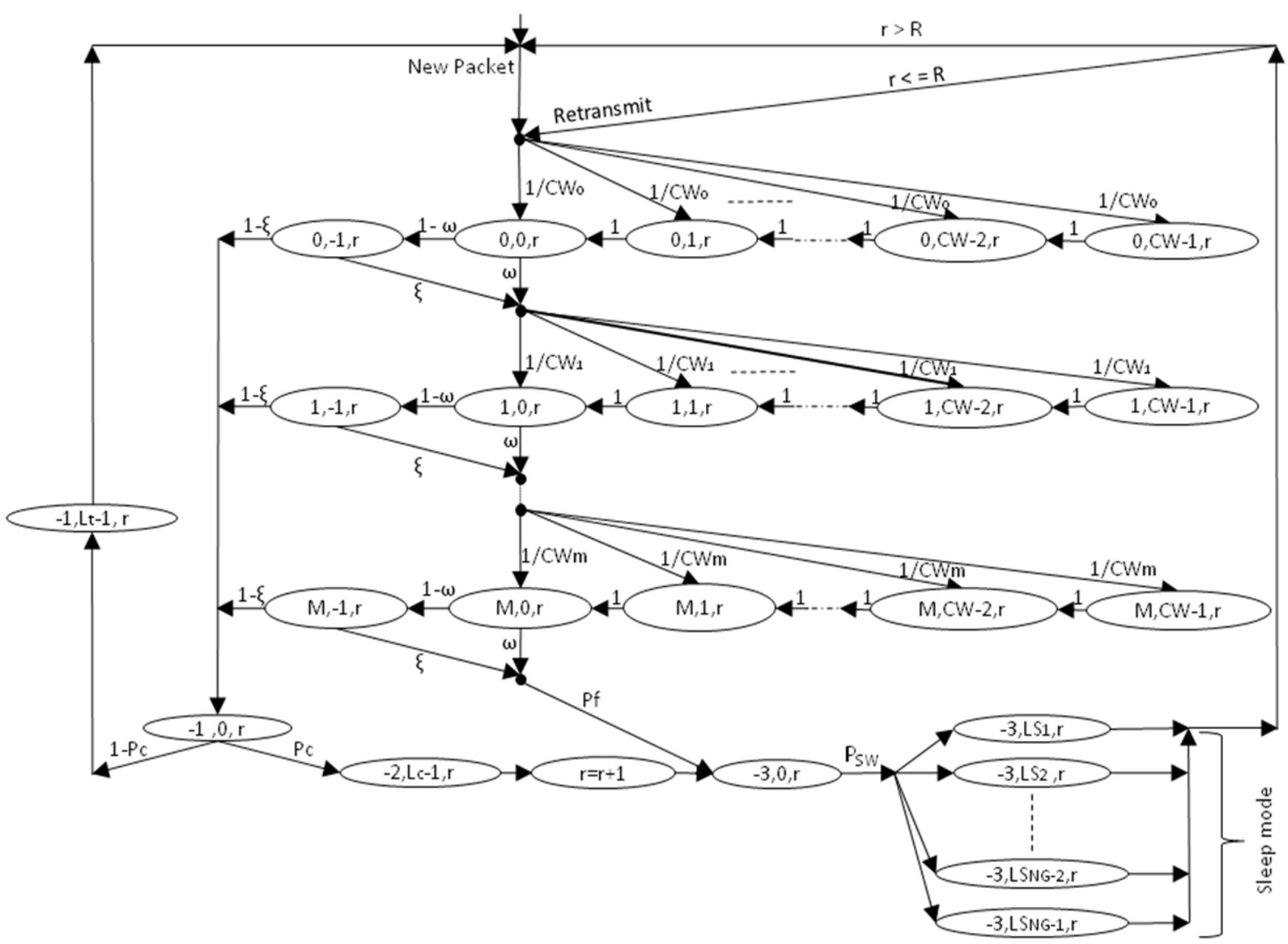

Fig. 5: Markov model for the proposed HSW-802.15.4

coupling is that the probability of sensing the carrier channel is constant and independent of other devices. Three stochastic processes describe the different states of the device during the transmission of a packet, namely: the backoff stage $G_{b}(t)$, the state of the backoff counter $G_{c}(t)$, and the state of the retransmission $G_{r}(t)$.

The integer time $(t)$ matches the beginning of the time slot and is declared as the delay stages (when $G_{b}(t) \in(0, N B F)$ or the transmission stage $\left(G_{r}(t)=-1\right)$. Furthermore, the stages $\left(G_{b}(t)=-2,-3\right)$ introduce the collision stage and the sleep/wake-up stage respectively. The $G_{c}(t)$ stage represents the delay counter of the device for a random duration between $(0$ and $C W i-1)$. The values $\left(G_{c}(t)=-1,-2\right)$ reflect to the first $C C A$ and second $C C A$ respectively. The parameters $M$ and $R$, in turn, are set to the values macMaxCSMABackoffs and macMaxFrameRetries. All other MAC parameters are shown in Table I. The probability $\omega$ refers to the likelihood of the channel assessment finding the channel busy during $C C A 1$, Whilst the probability $\xi$ pertains to channel assessment of finding the channel busy during $C C A 2$. The stages $(-1, k, j),(-2, k, j)$ and $(-3, k, j)$ characterise the transmission state, the packet collision state, and the dynamic sleep state respectively. The parameters $L_{s}$, and $L_{c}$ denote the durations of packet transmission and packet collision, respectively. The transition probabilities for the proposed three-dimensional Markov model can therefore be derived as follows:

$$
\begin{gathered}
P(i, j, r \mid i, j+1, r)=1, \text { for } j \geq 0 \\
P(i, j, r \mid i-1,0, r)=\frac{\omega+\xi-\omega \xi}{C W_{i}}, \text { for } i \leq M \\
P(0, j, 0 \mid i, 0, r)=\frac{1-\omega-\xi+\omega \xi}{C W_{0}}, \text { for } i \leq M, r<R \\
P(0, j, r \mid i, 0, r-1)=\frac{(1-\omega-\xi+\omega \xi) P_{S W}}{C W_{0}}, \text { forr }<R \\
P(0, j, 0 \mid M, 0, r)=\left[\frac{1-\omega-\xi+\omega \xi}{C W_{0}}\right. \\
+\frac{(1-\omega-\xi+\omega \xi) P_{S W}}{C W_{0}} \\
\left.+\frac{(\omega+\xi-\omega \xi) P_{S W}}{C W_{0}}\right], \text { forr }=R
\end{gathered}
$$


Equation (3) represents the delay counter for a random number of slots. Equation (4) provides the probability that the device picks a state in the next delay stage due to sensing the channel is busy during either the first or the second $C C A$, with probabilities $\omega$ and $\xi$, respectively. Equation (5) refers to the probability of successfully sending the packet without collision after sensing the channel is idle for both $C C A s$ and $(r<R)$. Equation (6) denotes the packet being sent for retransmission due to collision. In this case, the device should calculate the $L S_{i}$ before retransmitting it under the condition of $(r<R)$. Equation (7) defines the probability of beginning a new transmission when the device is in the final delay stage. This is either due to: sending the packet successfully; sending it with a collision after sensing the channel is idle for both $C C A s$, whereby the device needs to calculate $L S_{i}$ before starting a new transmission; or channel access failure during either $C C A 1$ or $C C A 2$, which means the device needs to calculate the $L S_{i}$ at a specific $T D M A_{\text {slot }}$.

The steady state probability for the proposed Markov model is denoted by $b_{i, j, r}=P_{r}\left\{\left(G_{b}(t), G_{c}(t), G_{r}(t)\right)=(i, j, r)\right\}$, where $i \in\{-3, M\}$, and $j \in\left\{-1, \max \left(C W_{i}-1, L_{t}-1, L_{c}-\right.\right.$ $\left.\left.1, L S_{i}-1\right)\right\}$, and $r \in\{0, R\}$.

$$
\begin{gathered}
b_{i, j, r}=\frac{C W_{i}-j}{C W_{i}} b_{i, 0, r} \\
b_{i, 0, r}=(\omega+(1-\omega) \xi)^{i} b_{0,0, r} \\
b_{0,0, r}=\left[P_{S W}\left(1-(\omega+\xi-\omega \xi)^{M+1}\right)\right]^{r} b_{0,0,0}
\end{gathered}
$$

From Equation(6), for $i=M$ and $r=R, b_{0,0,0}$ is located from the following

$$
\begin{aligned}
\sum_{i=0}^{M} \sum_{j=0}^{C W_{i}-1} & \sum_{r=0}^{R} b_{i, j, r}+\sum_{i=0}^{M} \sum_{r=0}^{R} b_{i, 0, r}+ \\
& \sum_{i=0}^{M} \sum_{r=0}^{R} b_{i,-1, r}+\sum_{j=0}^{L_{t}-1} \sum_{r=0}^{R} b_{-1, j, r}+ \\
& \sum_{j=0}^{L_{c}-1} \sum_{r=0}^{R} b_{-2, j, r}+\sum_{j=0}^{N G-1} \sum_{r=0}^{R} b_{-3, j, r}=1
\end{aligned}
$$

Next each expression in equation(11) is derived separately according to equations (8),(9) and (10). Furthermore, the delay line $C W_{i}$ is started with $C W_{0}=2^{M i n B E}$ and $C W_{M a x}=$ $2^{M a x B E}$, where $(\operatorname{MaxBE}-\operatorname{MinBE}=D i f) \geq i \leq M$ to get

$$
\begin{aligned}
\sum_{i=0}^{M} \sum_{j=0}^{C W_{i}-1} \sum_{r=0}^{R} b_{i, j, r}=\frac{b_{0,0,0}}{2}[\Gamma+\Psi \\
\times\left(C W_{0} \frac{1-(2(\omega+\xi-\omega \xi))^{D i f+1}}{1-2(\omega+\xi-\omega \xi)}\right. \\
\left.\left.+C W_{0} 2^{D i f} \frac{1-(\omega+\xi-\omega \xi)^{M}}{1-\omega-\xi+\omega \xi}\right)\right]
\end{aligned}
$$

and

$$
\begin{gathered}
\sum_{i=0}^{M} \sum_{r=0}^{R} b_{i, 0, r}+\sum_{i=0}^{M} \sum_{r=0}^{R} b_{i,-1, r} \\
+\sum_{j=0}^{L_{t}-1} \sum_{r=0}^{R} b_{-1, j, r}+\sum_{j=0}^{L_{c}-1} \sum_{r=0}^{R} b_{-2, j, r}= \\
\Gamma \times\left((2-\omega)+(1-\omega-\xi+\omega \xi)\left(L_{t}\left(1-P_{c}\right)+P_{c} L_{c}\right)\right) b_{0,0,0}
\end{gathered}
$$

where,

$$
\Psi=\frac{1-\left(P_{S W}\left(1-(\omega+\xi-\omega \xi)^{M+1}\right)\right)^{R+1}}{1-P_{S W}\left(1-(\omega+\xi-\omega \xi)^{M+1}\right)}
$$

and $\Gamma$ is equivalent to

$$
\Gamma \quad \Psi \quad \times \quad \frac{1-(\omega+\xi-\omega \xi)^{M+1}}{1-\omega-\xi+\omega \xi}
$$

For simplicity, we assume in the analysis that each $S G_{i}$ has a fixed value of sleep period $(L S)$. Then the last expression of equation (11) is expressed as

$$
\begin{gathered}
\sum_{j=0}^{N G-1} \sum_{r=0}^{R} b_{-3, j, r}=\frac{L S \times N G+L S}{2} \sum_{r=0}^{R} b(-3,0, r) \\
=\frac{L S \times N G+L S}{2} \times \\
\left(\left[L_{c} P_{c}+\left(1-L_{c} P_{c}\right)(\omega+\xi-\omega \xi)^{M+1}\right] \times \Psi\right) b_{0,0,0}
\end{gathered}
$$

$P_{S W}$ signifies the probability that the device is in the sleep stage and equivalent to:

$$
P_{S W}=L_{c} P_{c}+P_{f}
$$

$P_{f}$ is calculated in section VII-A, and $P_{c}$ which is the probability of more than one node that sense an idle channel and start their transmission at the same time. The $P_{c}$ is given by

$$
P_{c}=1-\frac{N \Theta(1-\Theta)^{N-1}}{1-(1-\Theta)^{N}}
$$

in which $\Theta$ is

$$
\Theta=\sum_{i=0}^{M-1} \sum_{r=0}^{R-1} b_{i, 0, r}
$$

and after analysing Equation 19 the value of $\Theta$ will be represented by $\left(\Gamma \times b_{0,0,0}\right)$. 


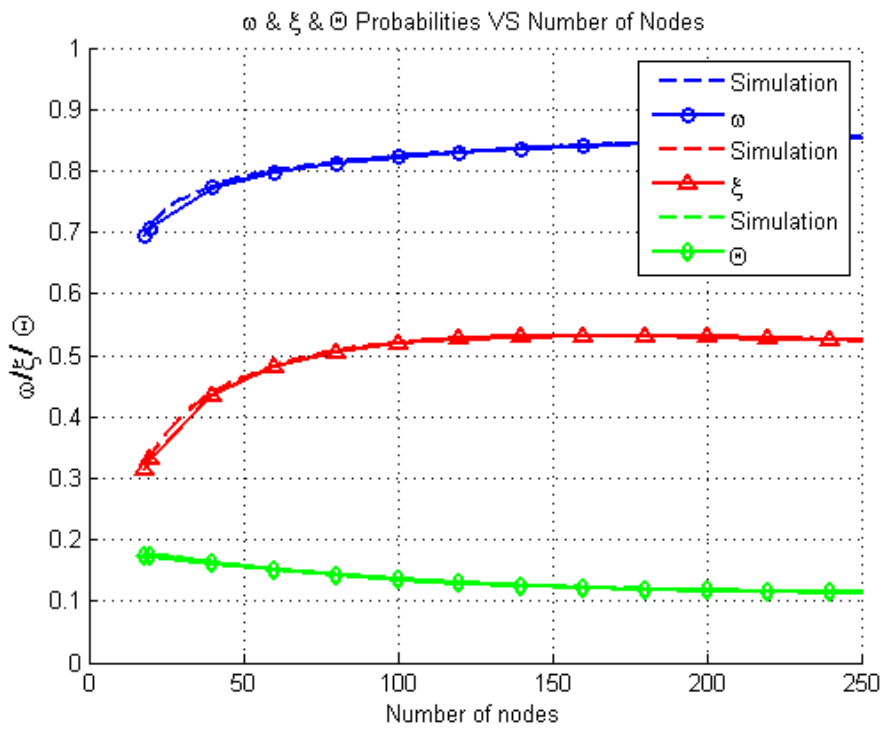

Fig. 6: This figure shows the validation of the proposed Markov model by comparing the numerical results of $\omega, \xi$ and $\Theta$ with the results obtained from the simulation when $N G=1$

\section{MOdel VALIDATION}

To confirm the accuracy of the proposed analytical model, Monte-Carlo simulations of the IEEE 802.15.4 basedcontention formulation are compared with the proposed model and included in the results. The simulation model attempts to imitate, as closely as possible, the actual transmission process based on the CSMA/CA of each device (See Appendix-B for more details about the simulation algorithm). In general, the CSMA/CA allows all the devices to compete for the channel access as a single group rather than as multiple ones. Hence, only $N G=1$ will be considered when validating the accuracy of the proposed model; however, all other $N G$ values have been provided to show the improvement in the proposed model over the traditional IEEE 802.15.4-based CSMA/CA protocol. The values of the parameters used to implement both the analytical model and the simulation runs are listed in Table I, and are identical to the default values of the IEEE 802.15.4 standard.

In Figure 6, the analytical model is compared to the simulation results with one value of $N G(N G=1)$ to validate the accuracy of the proposed model. This is because the proposed analytical model behaves identically to the traditional IEEE 802.15.4 standard when $N G=1$, which means there is no sleep mode and all the devices compete to access the channel as one group.

The figure presents the validation for the derived expressions $\xi, \omega$ and $\Theta$ when compared to the simulation results. As shown, the values of the analytical prediction results are accurate and very close to those of the simulation results. Additionally, when the number of devices is increased, the values of $\omega$ and $\xi$ increase, whilst the value of $\Theta$ decreases. Thus, there is an increase in the number of collisions and channel failures when the number of devices is increased.

Figure 7 displays the $\omega$ probability against the number of

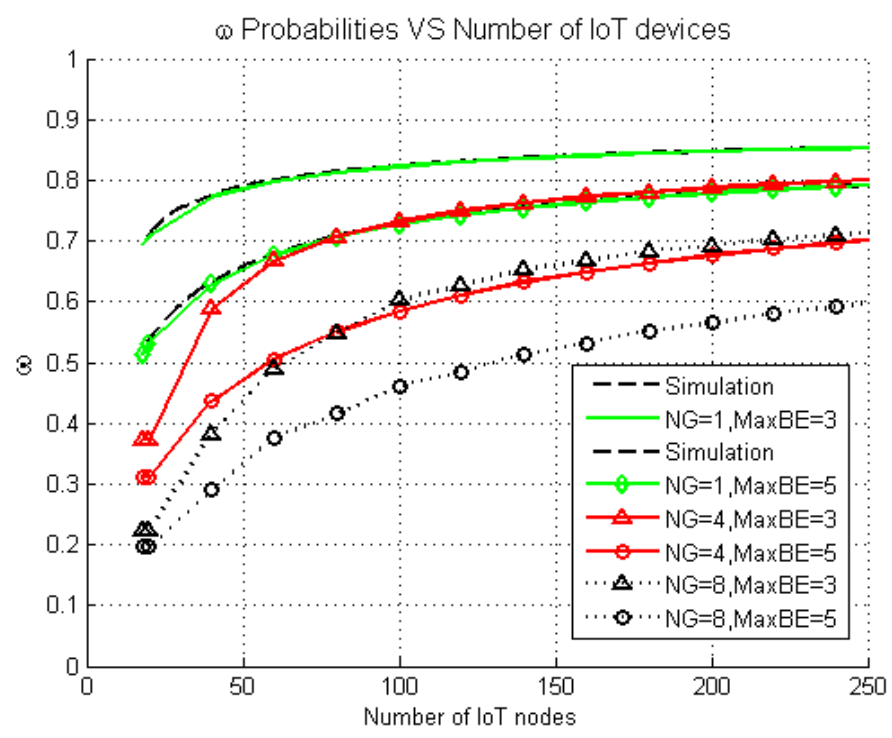

Fig. 7: $\omega$ validation for the proposed HSW-802.15.4 when $\operatorname{Max} B E=3$ and 5 and with different values of $N G$

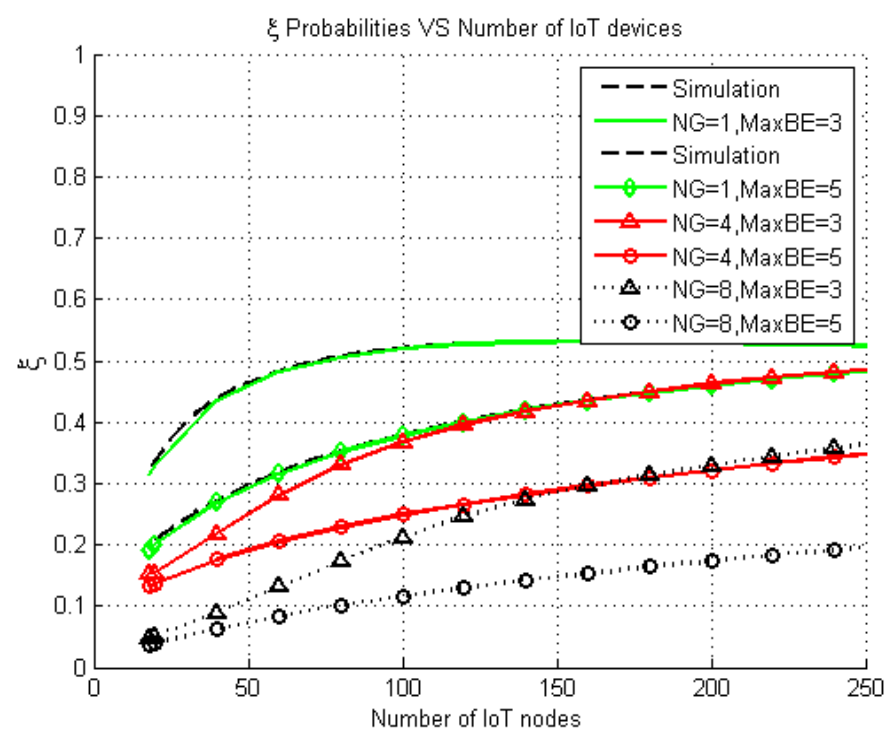

Fig. 8: $\xi$ validation for the proposed HSW-802.15.4 with different values of $N G$

devices. The value of $\omega$ has been tested when $N G=1,4$ and 8 , and $\operatorname{Max} B E=3$ and 5 . This figure shows that, during the channel sensing process, the HSW-802.15.4 performs the same, or better, when $N G=4$ or 8 , and $M a x B E=3$, than when $N G=1$ and $M a x B E=5$. This means that the value of $N G$ with lower power consumption due to sleep mode can compensate the $\operatorname{MaxBE}$ when the device consumes more power, especially in a high-density network. Figure 8 also shows the effect of the $N G$ and $\operatorname{MaxBE}$ on channel sensing for the second CCA with the probability $\xi$.

\section{Metrics Results}

In this section, the analytical model is used to describe numerically the performance of the HSW-802.15.4 model and the 
results from this are compared to those of the IEEE 802.15.4 standard in terms of: failure probability $\left(P_{f}\right)$, throughput $(S)$, channel utilisation $(\rho)$, and power consumption $\left(P_{W}\right)$. The performance of the proposed protocol has been examined with different numbers of subgroups $(N G=1 ; 2 ; 3 ; 4 ; 8$ and 16$)$. It should be noted that theproposed HSW-802.15.4 behaves identically to the IEEE 802.15.4 standard when $N G=1$.

\section{A. Failure Probability}

Failure probability $\left(P_{f}\right)$ refers to the probability of a packet being discarded as the result of either channel access failure (CAF) or retransmission limits (RL). According to the Markov model in Figure 5, the $P_{f}$ can be mathematically represented as follows:

$$
\begin{aligned}
& P_{f}=P_{R L}+P_{C A F} \\
&=\left[\left(P_{S W}\left(1-(\omega+\xi-\omega \xi)^{M+1}\right)\right)^{R+1}+\right. \\
& \\
&\left.\quad \Psi \times(\omega+\xi-\omega \xi)^{M+1}\right] b_{0,0,0}
\end{aligned}
$$

where, the $P_{C A F}$ and $P_{R L}$ are the probabilities of the packet getting discarded due to $C A F$ and $R L$, respectively.

Figure 9 shows the $P_{f}$ against the number of devices with different values of $N G$ and it is clear from the figure that failure probabilities decrease with higher values. This justifies the effectiveness of the proposed sleep/wakeup protocol, especially when the network begins to suffer from a higher number of collisions or channel failure. When the number of subgroups increase, the number of sleep devices also increases, which leads to a decrease in the number of contending devices during the $C P$ and consequently, the number of collisions fall and channel failure is more likely to be avoided.

\section{B. Throughput}

The throughput $(S($ bit/sec $))$ is defined as the aggregated amount of data that is successfully transmitted during a time specified. Successful transmission means that the device needs to sense the channel successfully for both CCAs and then send the packet without channel failure or collision. The $S$ can be mathematically represented as follows:

$$
S=D R \times \varphi
$$

where, $\varphi$ is calculated as follows:

$$
\varphi=\Theta(1-\omega)(1-\xi)\left(1-P_{S W}\right)
$$

where, $D R$ represents the data rate (see Table I). The throughput generated using the proposed model is explored using different values of $N G$. Figure 10 shows the throughput against different numbers of devices when $N G=1,2,3,4,8$ and 16. It shows that the throughput is increased when $N G$ is increased. However, the throughput starts decreasing with

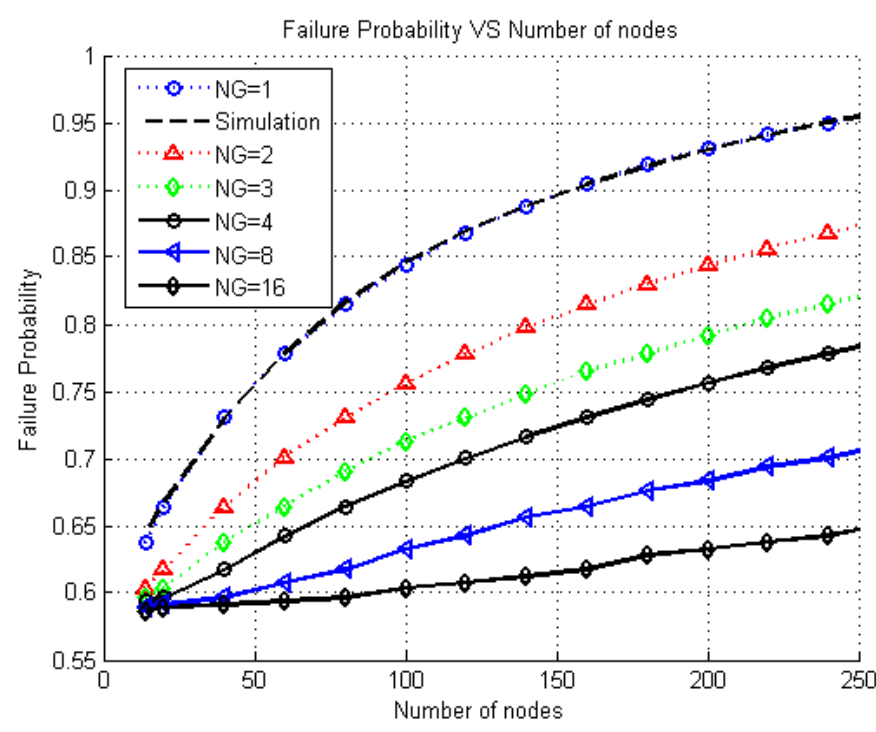

Fig. 9: Probability of failure $P_{F}$ with different values of NG and with MAC parameters: $\operatorname{Max} B E=5$ and $M=3$

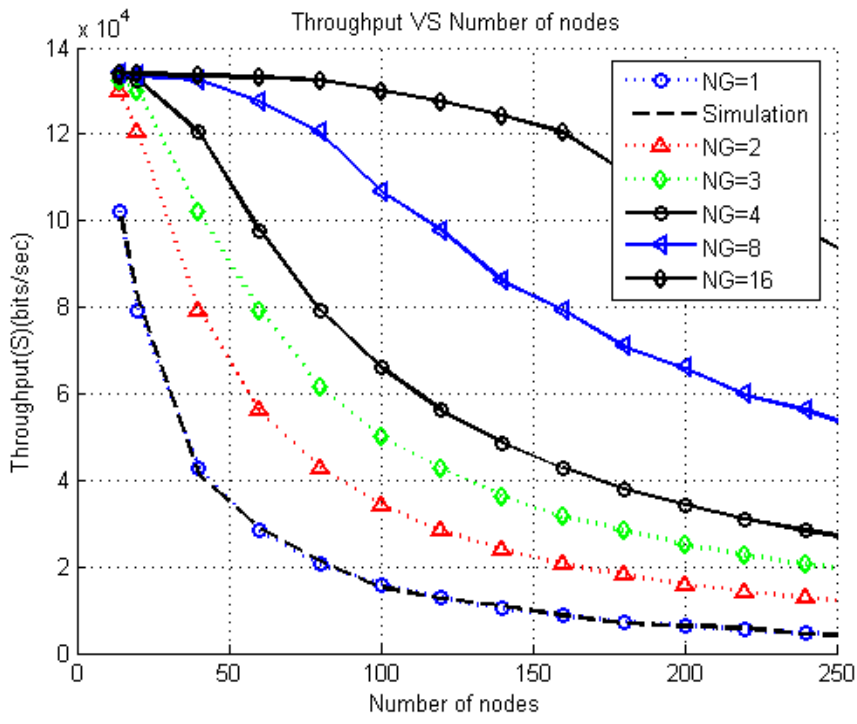

Fig. 10: Average throughput $S$ with different values of $N G$

all values of $N G$ when the number of devices increases, which is because the number of collisions starts to increase when there are more devices. To compensate, the proposed protocol expands to cover a larger number of devices in the network. In conclusion, there is an inverse relationship between the throughput and the number of devices, and vice versa depending on $N G$.

\section{Channel Utilisation}

The channel utilisation $(\rho)$ for one superframe is defined as the summation of the time during the successful transmission of packets to the total time during one superframe $\left(S F_{\text {duration }}\right)$. The $\rho$ in the simulation can be mathematically evaluated as: 


$$
\rho=\frac{\left(S F_{\text {duration }}-T_{B N}-T_{S T}\right) \times \varphi}{S F_{\text {duration }}}
$$

and $S F_{\text {duration }}$ can be clarified as

$$
S F_{\text {duration }}=\sum_{i=0}^{N_{s}} T D M A_{\text {slot }} i+T_{B N}+T_{S T}
$$

where, $N_{s}$ represents the number of TDM $A_{\text {slots }}, T_{B N}$ refers to the beacon notification interval. $T_{S T}$ represents the time required to broadcast the $S T$ message and it is comparable to $2 \times T_{B N}$. The value of $T_{B N}$, in the results, is taken equivalent to at least one $P C K_{L} . P C K_{L}$ refers to the total length of the packet including overhead and payload (see Table I). Then, $T_{B N}$ is estimated as

$$
T_{B N}=\left\lceil\frac{P C K_{L}}{D R}\right\rceil
$$

Figure 11 investigates $\rho$ in terms of the number of devices using different values of $N G$. As shown in the figure, the proposed HSW-802.15.4 provides a better $\rho$ when $N G=2,3,4,8$ or 16 . Moreover, the value of $\rho$ is high when the number of devices is less than 50 , for from there it then starts decreasing when the number of devices is increased. Finally, the figure also shows that the proposed protocol means that $\rho$ remains high even with a higher number of devices, which indicates the effectiveness of proposed protocol in a higher network density. Furthermore, the value of $\rho$ stays high for a longer time when $N G$ is increased.

\section{Delay}

The average delay $\left(D_{a v g}\right)$ is defined as the summation of the periods that the devices elapse in each transmission stage to obtain a successful one, i.e. receiving the ACK message. The transmission stages are: the backoff counter, channel sensing (CCAs), sleep/wake-up and packet transmission (either collision or successful). Hence, the $D_{\text {avg }}$ spent by the network during one entire superframe duration is equivalent to the summation of all the periods excluded the time during the successful transmission one. Then, it can be expressed as:

$$
D_{\text {avg }}=S F_{\text {duration }}-\left(S F_{\text {duration }}-T_{B N}-T_{S T}\right) \times \varphi
$$

where, the values of $\varphi, S F_{\text {duration }}$ and $T_{B N}$ are shown in equations 22,24 and 25 , respectively.

Figure 12 inspects $D_{\text {avg }}$ in terms of the number of devices and values of $N G=1,4,8$ and 16. The figure shows that the analytical results when $N G=1$ is well estimated in comparison with the simulation results. Furthermore, it reveals that the proposed HSW-802.15.4 protocol runs with a lower $D_{\text {avg }}$ when $N G=4,8$ or 16 than the IEEE802.15.4 standard. Moreover, as expected, the figure presents that $D_{\text {avg }}$ exponentially increases as the network density is grows and illustrates almost the same performance as the standard with a lower density network. Finally, the proposed protocol displays that $D_{\text {avg }}$ remains lower even with a higher number of devices,

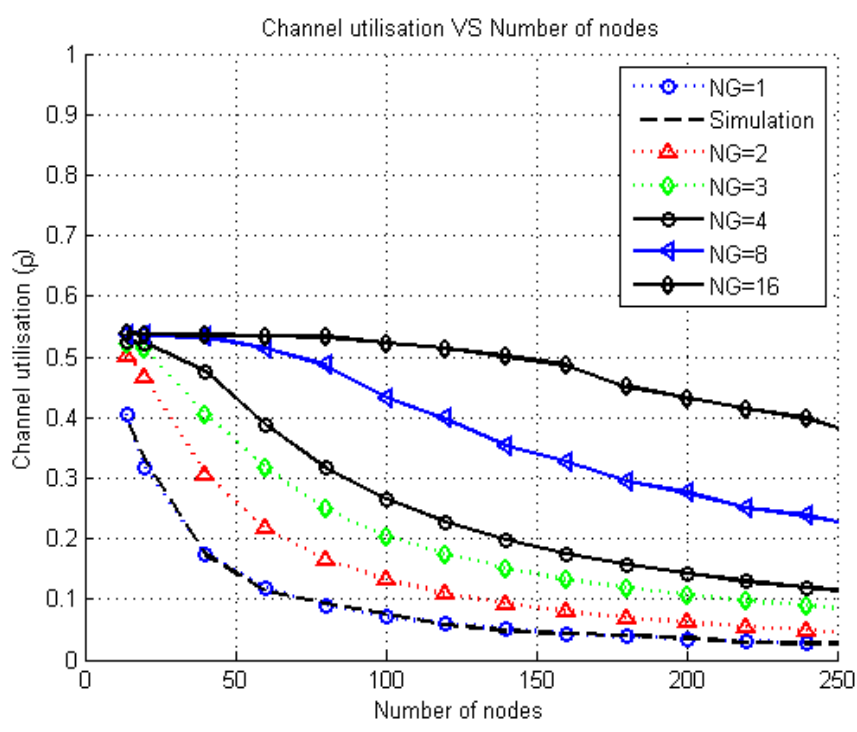

Fig. 11: Channel utilisation $\rho$ with different values of $N G$

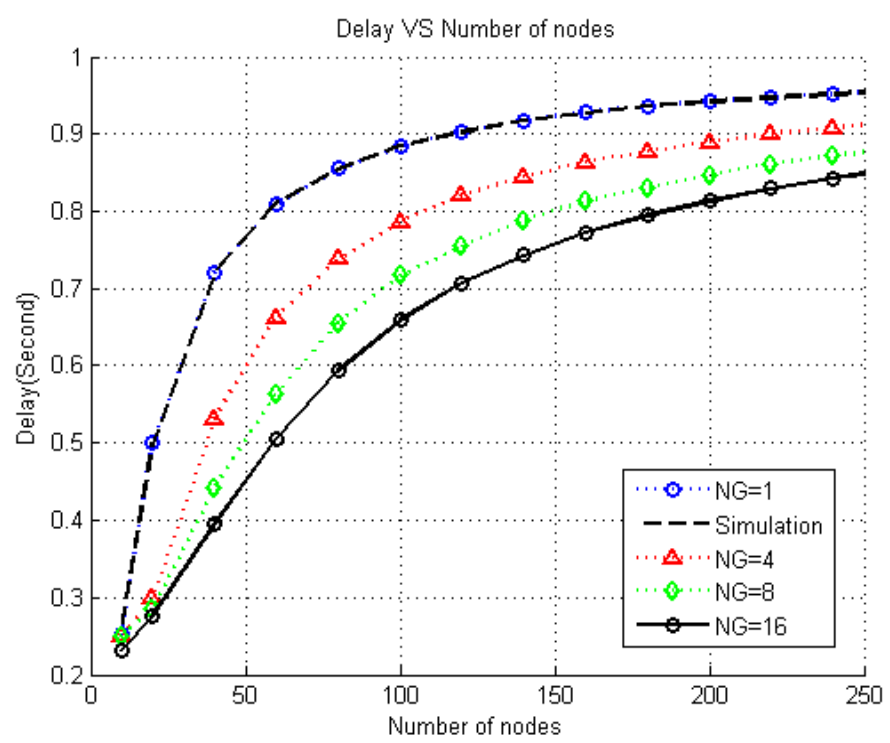

Fig. 12: Total average delay $\left(D_{\text {avg }}\right)$ with $N G$ values $=1,4,8$ and 16, and with MAC parameters: $M a x B E=5$ and $M=3$

which indicates its effectiveness with higher network density. This efficiency in the $D_{\text {avg }}$ metric is the result of increasing the network performance owing to device scheduling to share the channel and improvement in the number of successful transmissions.

\section{E. Power Consumption}

In this subsection the average energy consumed by a device attempting to transmit a single packet using the proposed HSW-802.15.4 protocol is considered. Using the IEEE 802.15.4 power parameters listed in Table I, the power consumed in idle, channel sensing, transmission, receiving and sleep modes is denoted by $P W_{\text {idle }}, P W_{C C A}, P W_{T x}, P W_{R x}$, and $P W_{S L}$, respectively. Furthermore, taking into consider- 
ation the Markov probabilities of the proposed protocol, the mean energy $E_{T o t}$ can be obtained as follows:

$$
\begin{aligned}
E_{T o t} & =P W_{i d l e} P r_{i d l e}+P W_{C C A} P r_{C C A} \\
& +P W_{T x} P r_{T x}+P W_{R x} P r_{R x}+P W_{S L} P r_{S L}
\end{aligned}
$$

where, $P r_{S L}$ is given in equation (16). $P r_{i d l e}$ is the probability of the device being idle either at the backoff, or at the time of waiting for the ACK $\left(L_{A C K-o u t}\right)$, and can be calculated as follows:

$$
P r_{i d l e}=\sum_{i=0}^{M} \sum_{j=0}^{C W_{i}-1} \sum_{r=0}^{R} b_{i, j, r}+\sum_{j=0}^{L_{A C K-o u t}} \sum_{r=0}^{R} b_{-2, j, r}
$$

$\operatorname{Pr}_{C C A}$ is the probability of channel sensing for two consecutive CCAs, and it is calculated as follows:

$$
\operatorname{Pr}_{C C A}=\Theta+(1-\omega) \Theta
$$

and $P r_{T x}$ is the transmission probability and calculated as:

$$
\operatorname{Pr}_{T x}=\frac{P C K_{L}}{D R} \times\left(1-(1-\Theta)^{N}\right)(1-\omega)(1-\xi)
$$

$\operatorname{Pr}_{R x}$ is the probability of successfully receiving the ACK after a transmission and it is given as follow:

$$
\operatorname{Pr}_{R x}=\sum_{j=0}^{L_{A C K}} \sum_{r=0}^{R} b_{-1, j, r}
$$

Figure 13 compares the energy consumed by the HSW802.15.4 MAC for the following values of $N G: 1,2,3,4,8$, and 16. This shows that average energy depletion has been improved by $40 \%$ to $60 \%$ with the proposed protocol depending on the number of devices and $N G$. This is because the devices avoid the high-energy depletion caused by attempting to access the channel and thus, there is no energy lost in idle stages waiting for the channel state to be detected, or when receiving unwanted packets due to collisions or channel overhead. Instead, the devices in the proposed protocol spend more time in a low energy sleep stage when network density is high and the $N G$ is increased, which results in a decrease in the number of competing devices depleting their energy in successful transmission or reception.

Figure 14 shows the energy consumed by the devices during the sleep stage. This shows that the device spends more energy on the sleep stage when the $N G$ value is increased. Energy consumption is, therefore, increased when the network density (number of devices) is increased. Furthermore, there is no energy consumption when $N G=1$, because there is no sleep stage, while the highest energy consumption occurs when $N G=16$. Thus, this figure illustrates why there is efficient utilisation of overall energy consumption, as the device consumes its energy during the sleep stage or in successful transmission and channel access, rather than in idle stages or higher energy depletion stages, such as collision or channel failure.

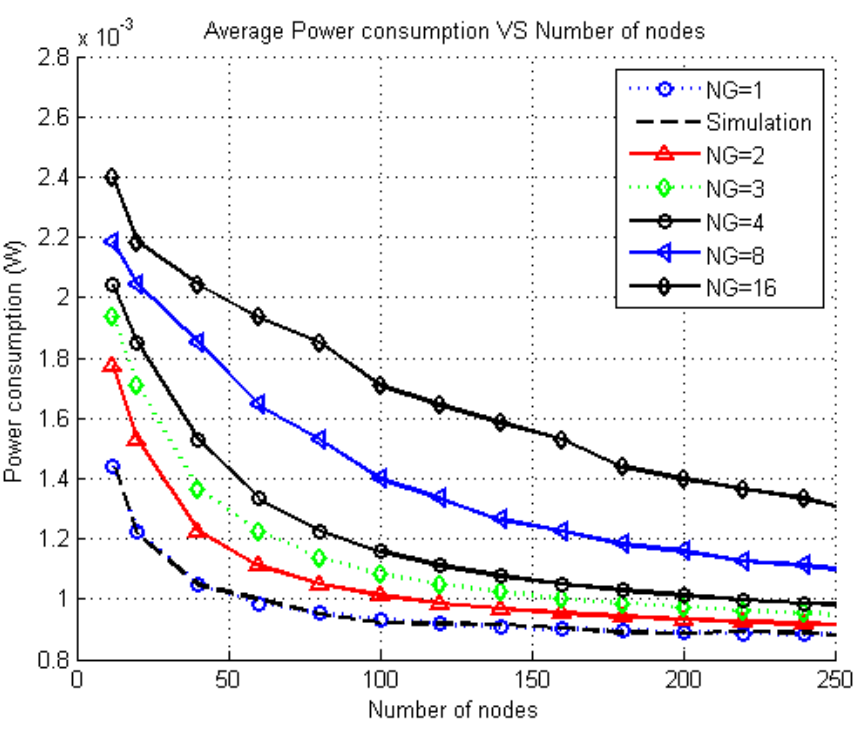

Fig. 13: The average power consumption with different values of $N G$ and $M a x B E=5$ as a function of saturated network

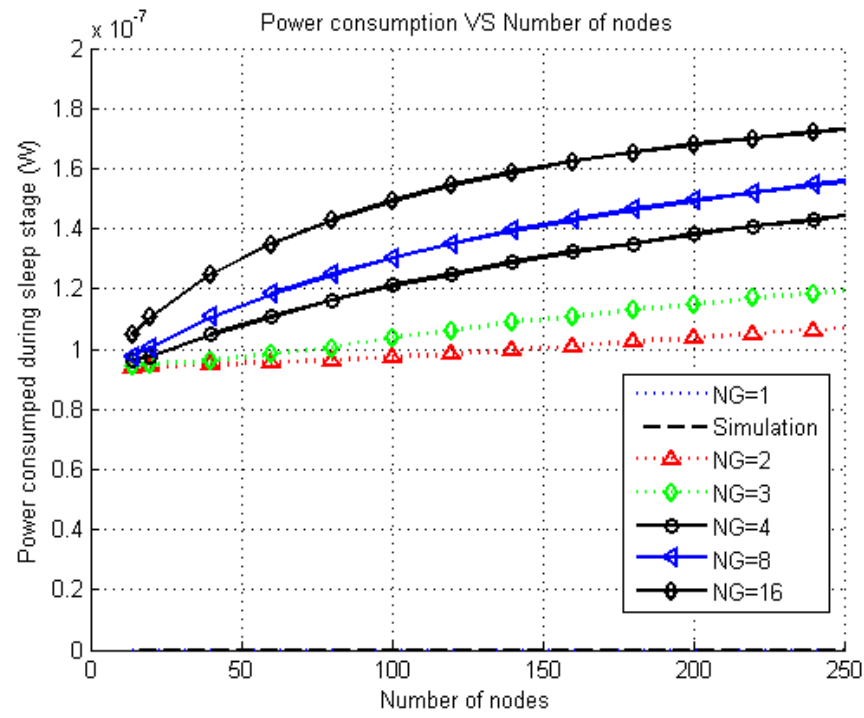

Fig. 14: Power consumption during the sleep stage with different values of $N G$

\section{CONCLUSION AND FUTURE WORK}

\section{A. Conclusion}

This study proposes a dynamic hybrid MAC protocol known as HSW-802.15.4, which adds an adaptable sleep/wake-up extension to the mechanism of the IEEE 802.15.4 standard. This hybrid MAC protocol enhances the channel access mechanism by merging the strong aspects of the CSMA/CA with those of the TDMA. The PAN coordinator broadcasts an ST message that categorises the network into $N G$ sleep groups of devices called $S G_{i}$. With the proposed extension, certain groups of devices are forced to sleep after each channel failure or collision. Each device that experiences channel collision or failure calculates its sleep period according to the scheduling 
information in the ST message and the TDMA $A_{\text {slot }}$ sequence. However, all the groups of devices are permitted to access the channel with low-density networks when there are no collisions or channel failures. Moreover, the number of IoT devices is increasing, and most of these are battery-powered. Hence, the proposed protocol enables IoT devices to conserve more power to access the channel, thereby prolonging the lifespan of the network regardless of the number of devices it contains. Moreover, dividing the devices into multiple sleep groups reduces the hidden node problem. A frame structure for the proposed protocol compatible with the IEEE 802.15.4 standard has also been developed. To provide a mathematical model for the proposed protocol, a theoretical analysis-based Markov chain has been constructed. The results show that the proposed HSW-802.15.4 protocol can efficiently utilise the energy of the battery by $40 \%$ to $60 \%$ depending on the $N G$, and this demonstrates very efficient channel utilisation with high throughput when compared to the performance of the IEEE 802.15.4 standard.

\section{B. Future Work}

The IEEE 802.15.4 MAC is subject to many other issues that also need to be investigated and incorporated to improve the energy conservation of the devices, some of these being the: back-off methodology, optimal duty cycle, unreliability, network complexity, packet size limitation and the hidden node problem. Finding efficient algorithms for these issues can prevent the high energy consumed by the devices, due to data transmission, reception, collision as well as the idle and sleep modes.

For instance, tuning the values of the maximum and minimum number of binary exponential back-off in an effective way will have a direct impact on the energy efficiency of any device. This is considered to be a better solution as will have a greater impact on the communication reliability, rather than increasing the number of retransmissions, for this will require higher energy consumption. Furthermore, depending on the application constraints and specifications, some devices are allocated a fixed duty cycle (long or short period), while others require adaptable periods. These two factors can obviously affect the unreliability issue in IEEE 802.15.4 MAC. This is because it employs the CSMA/CA mechanism to access the channel, which is considered as being an inefficient algorithm for controlling the number of collisions, especially in high density networks.

Another important solution to conserving the energy consumed by IoT devices and reducing the network complexity is the involvement of SDN and network virtualisation technologies in the MAC process. These can be implemented in different ways, such as proactively, reactively (on demand) or by mixing between the two. With these approaches, the SDN controller has the responsibility of monitoring the number of network traffic collisions and deciding upon the optimal sleep/wake-up period for each device in a way that eliminates the effect of the hidden node issue. These methods can be easily combined with the proposed HSW-802.15.4 MAC protocol, because the SDN controller and the virtualisation technology over the cloud can categorise the devices into several groups and identify the TDMA time slots as well as the group associated with each. Furthermore, the involvement of such technologies in the MAC protocol will facilitate the protocol updating process, according to the network requirements, such as in the case of an emergency. In this case, the device will need to be accompanied by the GPS facility in order to be able to send an emergency request, when required, to the SDN controller over the cloud, which in turn will update the network with an emergent route for the requested device.

\section{APPENDIX}

\section{A. Notation}

The notation used throughout this paper are described in Table III.

TABLE III: The notation used throughout this paper

\begin{tabular}{|l|l|}
\hline Symbol & Description \\
\hline$N$ & Number of IoT devices in the network \\
\hline$\omega$ & Probability of start sensing the channel during the first CCA \\
\hline$\xi$ & Probability of finding the channel busy during the first CCA \\
\hline$C W$ & Probability of finding the channel busy during the second CCA \\
\hline$L S_{i}$ & Number of $T D M A_{\text {slots }}$ sleep for $S G_{i}$ \\
\hline$p_{s w}$ & Probability of sleep/wake-up \\
\hline$P_{f}$ & probability of failure \\
\hline$P_{C}$ & Probability of collision \\
\hline$S$ & Network Throughput \\
\hline$\rho$ & Channel utilisation \\
\hline$D_{a v g}$ & Average delay \\
\hline$P W$ & Power consumption \\
\hline$P_{R L}$ & Probability of the packet getting discarded due to RL \\
\hline$P_{C A F}$ & Probability of the packet getting discarded due to CAF \\
\hline
\end{tabular}

\section{B. CSMA/CA Algorithm}

Algorithm-1 describes the behaviour of the CSMA/CA mechanism, which is similar to the simulator used by [14] with some enhancements. After guaranty the accuracy of the simulator by gotten the same results presented by [14], its results have been compared with the proposed numerical model. Furthermore, the notation used in Algorithm-1 have been described in Table I and Table II (in Appendix-A). 


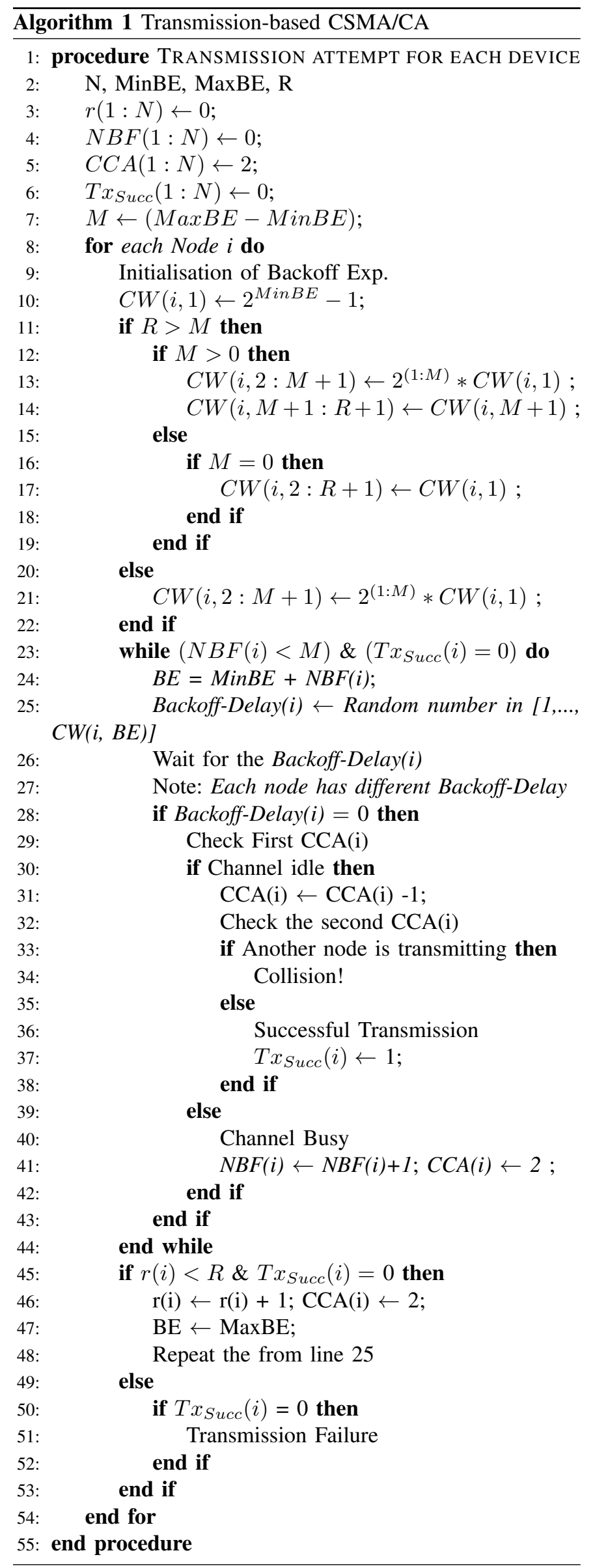

\section{REFERENCES}

[1] Y. Liu, C. Yuen, J. Chen, and X. Cao, "A scalable hybrid mac protocol for massive $\mathrm{m} 2 \mathrm{~m}$ networks," in Wireless Communications and Networking Conference (WCNC), 2013 IEEE. IEEE, 2013, pp. 250255.

[2] A. Al-Fuqaha, M. Guizani, M. Mohammadi, M. Aledhari, and M. Ayyash, "Internet of things: A survey on enabling technologies, protocols, and applications," IEEE Communications Surveys \& Tutorials, vol. 17, no. 4, pp. 2347-2376, 2015.

[3] A. Aijaz and A. H. Aghvami, "Cognitive machine-to-machine communications for internet-of-things: A protocol stack perspective," IEEE Internet of Things Journal, vol. 2, no. 2, pp. 103-112, 2015.

[4] IEEE 802.15.4-2006 (Revision of IEEE Std 802.15.4-2003), "IEEE Standard for Information Technology - Local and Metropolitan Area Networks - Specific Requirements - Part 15.4: Wireless Medium Access Control (MAC) and Physical Layer (PHY) Specifications for Low Rate Wireless Personal Area Networks (WPANs)," https://standards.ieee.org, 2006, [Online; accessed 1-March-2016].

[5] B. Bandyopadhyay, S. J. Ahmed, D. Das, and A. Chatterjee, "As 802.15. 4: A modified ieee 802.15. 4 standard for more reliable communication and utilization of inactive period using optimized sleep period," in India Conference (INDICON), 2014 Annual IEEE. IEEE, 2014, pp. 1-6.

[6] C. G. Peces, J. Eriksson, and N. Tsiftes, "Sleepy devices vs. radio duty cycling: The case of lightweight $\mathrm{m} 2 \mathrm{~m}$," IEEE Internet of Things Journal, 2018.

[7] A. Rajandekar and B. Sikdar, "A survey of mac layer issues and protocols for machine-to-machine communications," IEEE Internet of Things Journal, vol. 2, no. 2, pp. 175-186, 2015.

[8] O. Tickoo and B. Sikdar, "Modeling queueing and channel access delay in unsaturated ieee 802.11 random access mac based wireless networks," IEEE/ACM Transactions on Networking (TON), vol. 16, no. 4, pp. 878$891,2008$.

[9] B. Shrestha, E. Hossain, and K. W. Choi, "Distributed and centralized hybrid csma/ca-tdma schemes for single-hop wireless networks," IEEE Transactions on Wireless Communications, vol. 13, no. 7, pp. 40504065, 2014.

[10] R. Bruno, M. Conti, and E. Gregori, "Optimal capacity of p-persistent csma protocols," IEEE communications letters, vol. 7, no. 3, pp. 139141, 2003.

[11] G. Bianchi, "Performance analysis of the ieee 802.11 distributed coordination function," IEEE Journal on selected areas in communications, vol. 18 , no. 3 , pp. $535-547,2000$.

[12] S. Pollin, M. Ergen, S. C. Ergen, B. Bougard, L. Van der Perre, I. Moerman, A. Bahai, P. Varaiya, and F. Catthoor, "Performance analysis of slotted carrier sense ieee 802.15. 4 medium access layer," IEEE Transactions on wireless communications, vol. 7, no. 9, 2008.

[13] V. Nguyen, T. Z. Oo, P. Chuan, and C. S. Hong, "An efficient time slot acquisition on the hybrid tdma/csma multichannel mac in vanets," IEEE Communications Letters, vol. 20, no. 5, pp. 970-973, 2016.

[14] A. Faridi, M. R. Palattella, A. Lozano, M. Dohler, G. Boggia, L. A. Grieco, and P. Camarda, "Comprehensive evaluation of the ieee 802.15. 4 mac layer performance with retransmissions," IEEE Transactions on Vehicular Technology, vol. 59, no. 8, pp. 3917-3932, 2010.

[15] P. K. Sahoo and J.-P. Sheu, "Modeling ieee 802.15.4 based wireless sensor network with packet retry limits," in Proceedings of the 5th ACM symposium on Performance evaluation of wireless ad hoc, sensor, and ubiquitous networks. ACM, 2008, pp. 63-70.

[16] P. Park, P. Di Marco, P. Soldati, C. Fischione, and K. H. johansson, "A generalized markov chain model for effective analysis of slotted ieee 802.15.4," in Mobile Adhoc and Sensor System, 2009. MASS'09. IEEE 6th International Conference on. IEEE, 2009, pp. 130-139.

[17] M.-H. Zayani, V. Gauthier, and D. Zeghlache, "A joint model for ieee 802.15. 4 physical and medium access control layers," in Wireless Communications and Mobile Computing Conference (IWCMC), 2011 7th International. IEEE, 2011, pp. 814-819.

[18] M. Zuniga and B. Krishnamachari, "Analyzing the transitional region in low power wireless links," in Sensor and Ad hoc Communications and Networks, 2004. IEEE SECON 2004. 2004 First Annual IEEE Communications Society Conference on. IEEE, 2004, pp. 517-526.

[19] B. Bandyopadhyay, S. J. Ahmed, M. K. Naskar, and A. Mukherjee, "Improving the performance metrics of beacon enabled ieee 802.15. 4 using adaptive synchronization algorithm for reliable communication," in Advanced Networks and Telecommuncations Systems (ANTS), 2013 IEEE International Conference on. IEEE, 2013, pp. 1-6. 
[20] Y. Zhuang and L. Ma, "An energy-efficient and low-collision ieee 802.15. 4-based mac for data gathering in wireless sensor networks," in Computer Science \& Service System (CSSS), 2012 International Conference on. IEEE, 2012, pp. 1244-1247.

[21] J. Mišic and V. B. Mišic, "Queuing analysis of sleep management in an 802.15.4 beacon enabled pan," in IEEE/ACM First International Workshop on Broadband Wireless Services and Applications (BroadWISE'04). IEEE, 2004.

[22] C. F. Chiasserini and M. Garetto, "An analytical model for woreless sensor networks with sleeping nodes," IEEE Transactions on Mobile Computing, vol. 5, no. 12, pp. 1706-1718, 2006.

[23] M. F. Ghazvini, M. Vahabi, M. Rasid, and A. R. Raja, "Energy efficiency in mac 802.15.4 for wireless sensor networks," in Telecommunication Technolohies 2008 and 2008 2nd Malaysia Conference on Photonics. NCTT-MCP 2008. 6th National Conference on. IEEE, 2008, pp. 289294.

[24] Z. Xiao, C. He, and L. Jiang, "Slot-based model for ieee 802.15. 4 mac with sleep mechanism," IEEE Communications Letters, vol. 14, no. 2, 2010.

[25] R. Jurdak, A. G. Ruzzelli, and G. M. O 'Hare, "Radio sleep mode optimisation in wireless sensor networks," IEEE Transactions on Mobile Computing, vol. 9, no. 7, pp. 955-968, 2010.

[26] M. Khanafer, M. Guennoun, and H. T. Mouftah, "Adaptive sleeping periods in ieee 802.15. 4 for efficient energy savings: markov-based theoretical analysis," in Communications (ICC), 2011 IEEE International Conference on. IEEE, 2011, pp. 1-6.

[27] Z. Xiao, C. He, and L. Jiang, "An analytical model for ieee 802.15. 4 with sleep mode based on time-varying queue," in Communications (ICC), 2011 IEEE International Conference on. IEEE, 2011, pp. 1-5.

[28] B. Shrestha, E. Hossain, and S. Camorlinga, "Ieee 802.15.4 mac with gts transmission for heterogeneous devices with application to wheelchair body area sensor networks," IEEE Transactions in Information Technology in Biomedicine, vol. 15, no. 5, pp. 767-777, 2011.

[29] S.-T. Sheu, Y.-Y. Shih, and W.-T. Lee, "Csma/cf protocol for ieee 802.15.4 wpans," IEEE Transactions on vehicular technology, vol. 58 no. 3, pp. 1501-1516, 2009.

[30] T. A. Al-Janabi and H. S. Al-Raweshidy, "A centralised routing protocol with a scheduled mobile sink-based ai for large scale i-iot," IEEE Sensors Journal, 2018.

[31] H. Ayadi, A. Zouinkhi, T. Val, A. van den Bossche, and M. N. Abdelkrim, "Network lifetime management in wireless sensor networks," IEEE Sensors Journal, 2018.

[32] N. Abramson, "The aloha system: another alternative for computer communications," in Proceedings of the November 17-19, 1970, fall joint computer conference. ACM, 1970, pp. 281-285.

[33] L. G. Roberts, "Aloha packet system with and without slots and capture," ACM SIGCOMM Computer Communication Review, vol. 5, no. 2, pp. 28-42, 1975.

[34] L. Kleinrock and F. Tobagi, "Packet switching in radio channels: Part i-carrier sense multiple-access modes and their throughput-delay characteristics," IEEE transactions on Communications, vol. 23, no. 12, pp. 1400-1416, 1975.

[35] I. Rhee, A. Warrier, M. Aia, J. Min, and M. L. Sichitiu, "Z-mac: a hybrid mac for wireless sensor networks," IEEE/ACM Transactions on Networking (TON), vol. 16, no. 3, pp. 511-524, 2008.

[36] R. Zhang, R. Ruby, J. Pan, L. Cai, and X. Shen, "A hybrid reservation/contention-based mac for video streaming over wireless networks," IEEE Journal on Selected Areas in Communications, vol. 28 , no. $3,2010$.

[37] M. Salajegheh, H. Soroush, and A. Kalis, "Hymac: Hybrid tdma/fdma medium access control protocol for wireless sensor networks," in Personal, Indoor and Mobile Radio Communications, 2007. PIMRC 2007. IEEE 18th International Symposium on. IEEE, 2007, pp. 1-5.

[38] T. Shu and M. Krunz, "Energy-efficient power/rate control and scheduling in hybrid tdma/cdma wireless sensor networks," Computer Networks, vol. 53, no. 9, pp. 1395-1408, 2009.

[39] M. Chatterjee and S. K. Das, "Performance evaluation of a requesttdma/cdma protocol for wireless networks," Journal of Interconnection Networks, vol. 2, no. 01, pp. 49-67, 2001.

[40] Y. Liu, C. Yuen, X. Cao, N. U. Hassan, and J. Chen, "Design of a scalable hybrid mac protocol for heterogeneous $\mathrm{m} 2 \mathrm{~m}$ networks," IEEE Internet of Things Journal, vol. 1, no. 1, pp. 99-111, 2014.

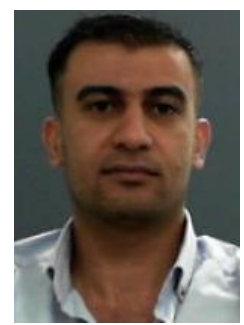

Thair A. Al-Janabi received the B.Sc. and M.Sc. degrees in information and communication engineering from Nahrain University, Baghdad, Iraq, in 2008 and 2011 respectively. He is currently working toward the $\mathrm{PhD}$ degree in wireless network engineering at Brunel University London, UK. His research interests are IoT, artificial intelligence, wireless sensor networks, development and optimization of routing and MAC protocols, SDN, NFV, and cloud computing.

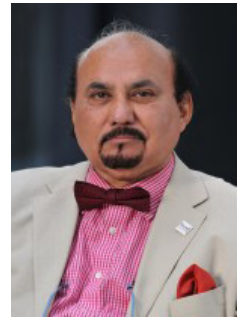

Hamed S. Al-Raweshidy awarded BEng and MSc from University of Technology, Baghdad in 1977 and 1980 respectively. He completed his Post Graduate Diploma from Glasgow University, Glasgow, UK in 1987. He was awarded his PhD in 1991 from Strathclyde University in Glasgow, UK. He has worked with The Space and Astronomy Research Centre (Iraq), PerkinElmer (USA), British Telecom (UK), Oxford University, Manchester Met. University, Kent University and currently he is the Director of Wireless Network Communications Centre (WNCC), Brunel University London, United Kingdom. 